\title{
Target normal sheath acceleration of protons using triple-layer target with long preplasma
}

\author{
Sheng-Fei Tong, Zheng-Mao Sheng, ${ }^{*}$ and M. Y. Yu \\ Institute for Fusion Theory and Simulation and Department of Physics, Zhejiang University, \\ Hangzhou 310027, China
}

(Received 16 January 2018; published 31 May 2018)

\begin{abstract}
Target-normal sheath acceleration (TNSA) of monoenergetic proton bunches using a triple-layer target consisting of a long slightly-above-critical-density carbon preplasma, a thin solid-density carbon main target layer, and a thin hydrogen layer attached to its back, is considered. The target-back space-charge field the consists of mainly the preplasma electrons that have been accelerated and heated by the relativistic laser pulse. The backside electron cloud is thus denser and more favorably profiled for TNSA of the protons from the hydrogen layer. It is found that a proton beam with a $\sim 460 \mathrm{MeV}$ monoenergetic peak energy can be generated. The corresponding energy and angular spread are $\sim 33 \mathrm{MeV}$ and $\sim 3^{\circ}$, respectively.
\end{abstract}

DOI: 10.1103/PhysRevAccelBeams.21.051303

\section{INTRODUCTION}

Laser-plasma interaction (LPI) can generate high-energy ion beams [1,2]. Potential applications of the latter include fast ignition of inertial fusion plasmas [1-3], cancer therapy [4,5], proton imaging [6], creation of rare isotopes, etc. LPI schemes for ion acceleration include target normal sheath acceleration (TNSA) [7-11], radiation pressure acceleration (RPA) [12-18], collisionless electrostatic shock acceleration [19-21], breakout afterburner acceleration [22], etc.

TNSA has been widely investigated for its relatively low demand on the laser and target parameters [7-9]. In TNSA the energetic electrons generated from the front target surface by the laser impact and accelerated forward can easily penetrate through the target and enter the backside vacuum, creating there an electrostatic space-charge, or sheath, field that can reach $\sim 10^{12} \mathrm{~V} / \mathrm{m}$. Ions on the rear target surface can thus be accelerated to high energies by this field. The scheme can also be enhanced by using a double-layer target consisting of a heavy-ion slab with a thin light-ion (say proton) layer attached to its back surface, thereby leading to more hot electrons and stronger sheath field, and thus more energetic TNSA protons. Recently, it has been shown that with a hybrid RPA-TNSA scheme, where a double-layer target is irradiated by an ultrashort ultraintense laser pulse, even more energetic proton bunches with less energy spread can be achieved [17].

\footnotetext{
*zmsheng@zju.edu.cn
}

Published by the American Physical Society under the terms of the Creative Commons Attribution 4.0 International license. Further distribution of this work must maintain attribution to the author(s) and the published article's title, journal citation, and DOI.
In the presence of preplasma generated by the laser prepulse, the electrons in the preplasma are also accelerated and heated by the main laser pulse and can easily propagate through the target and enter the backside vacuum [23-27]. Similarly, a low-density or foam layer in front of the main target layer can enhance the density and energy of the hot electrons producing the sheath field behind the target [5,28-30].

In this paper, we investigate TNSA from the interaction of an intense laser pulse with a triple-layer target consisting of a long suitable carbon foam layer as slightly over-critical density (SOCD) preplasma, a thin main soliddensity carbon target layer, and a thin proton layer. Twodimensional(2D) particle-in-cell (PIC) simulations show that as the radiation pressure of the leading front of the laser pulse accelerates and expels preplasma electrons, a channel of much reduced electron density is formed. The electrons remaining in the channel are then accelerated by the trailing part of the laser. They easily pass through the thin highdensity main target and proton layers and enter the backside vacuum. The resulting intense space-charge field there can efficiently accelerate the protons in the proton layer as well as prevent the lower-energy target electrons from entering the region. It is found that, as shown in Fig. 1, high-energy protons with low spreads in energy as well as spatial angle can be produced.

\section{TNSA OF PROTONS}

A linearly polarized Gaussian laser pulse of wavelength $\lambda=1 \mu \mathrm{m}$, waist $w=6 \lambda$, and laser parameter $a_{0}=e E_{0} / m_{e} \omega c=30 \quad$ (or peak intensity $I_{0}=1.23 \times$ $10^{21} \mathrm{~W} / \mathrm{cm}^{2}$ ), where $-e$ and $m_{e}$ are the electron charge and mass, respectively, $\omega$ is the laser frequency, and $E_{0}$ is the peak laser electric field, irradiates normally from the left of 

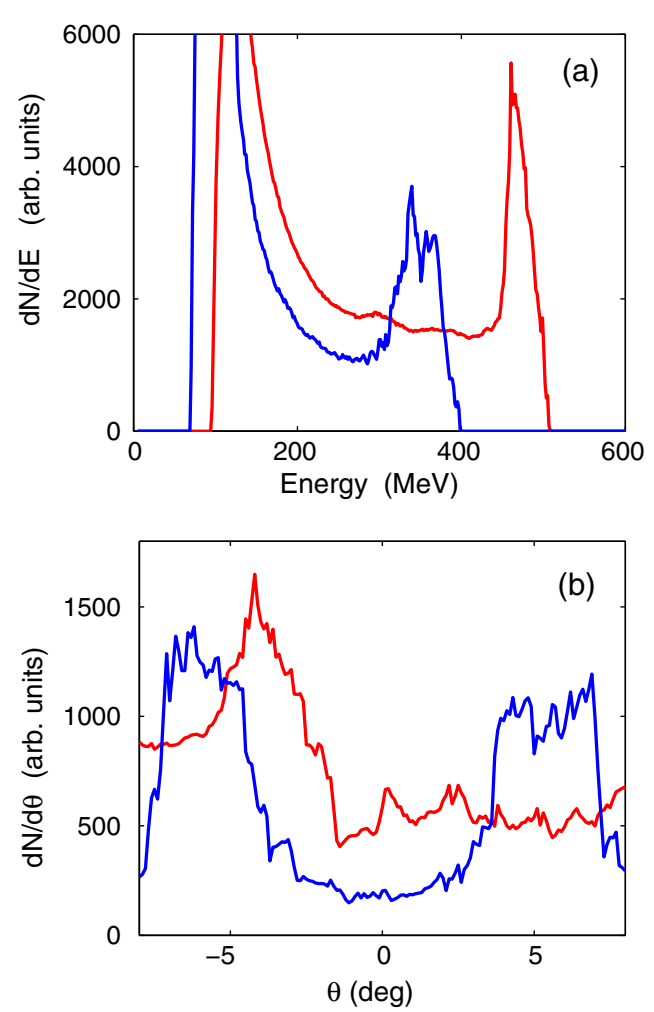

FIG. 1. (a) Energy and (b) angle spectra of the TNSA protons behind the target at $t=200 T$. The red and blue curves are for the cases with and without the preplasma, respectively. One can see that the preplasma leads to higher energy, narrower and singlepeaked angular distribution, as well as more monoenergetic protons. The laser axis is at $\theta=0$.

the simulation box in the $x$ direction. The envelope of the laser electric field is

$$
E_{y}(x, y, t)=a_{0} \sqrt{\frac{w_{0}}{w(x)}} e^{-\frac{\left(y-y_{0}\right)^{2}}{w^{2}(x)}} e^{-\frac{1}{2}\left(\frac{(t-2 L}{L}\right)^{2}},
$$

where $w^{2}(x)=w_{0}^{2}\left[1+\left(x / x_{0}\right)^{2}\right], L=\tau / 1.665$, and $\tau=40 T$.

The fully ionized triple-layer target consists of a $0.5 \lambda$ thick dense carbon layer placed at $x=30 \lambda$. It is preceded by a $27 \lambda$-thick carbon preplasma that starts at $x=3 \lambda$. Attached to its back is a $0.05 \lambda$-thin hydrogen layer as the proton source. The lateral ( $y$ direction) width of the target is $60 \lambda$. The electron densities of the SOCD carbon preplasma, the thin dense carbon main target layer, and the hydrogen layers are $1.8 n_{c}, 30 n_{c}$, and $1 n_{c}$, respectively, where $n_{c}=$ $1.11 \times 10^{21} \mathrm{~cm}^{-3}$ is the critical density. Thus, the densities of the carbon ions in the preplasma and the main layers are $0.3 n_{c}$ and $5 n_{c}$, respectively. The simulation box is $120 \mu \mathrm{m} \times$ $80 \mu \mathrm{m}$ and it has $12000 \times 2000$ cells, with 100 simulation particles per cell for the carbon and proton layers, and 16 particles per cell for the carbon preplasma.

As the laser propagates in the SOCD preplasma, the affected electrons are heated and driven forward as well as radially outward, and the laser becomes self-focused
[31-33], creating a positively charged channel containing mainly carbon ions. The forward accelerated hot electrons can easily enter the target-back vacuum. The electrons in the preplasma are also driven forward directly by the relativistic electric and magnetic field oscillations, as shall be discussed in more detail below. They move with the laser pulse in the preplasma and can pass through the rest of the target into the rear vacuum, enhancing the sheath field there. As the laser light reaches the thin dense carbon layer in the middle, it is reflected and electrons in the front surface of the latter are also pulled out, heated, and accelerated forward. However, in the present scheme the hot electrons in the target-backside sheath are predominantly from the preplasma. Such a TNSA regime, where RPA as well as direct laser acceleration of electrons take place in the preplasma, does not seem to have been investigated previously.

Figure 1 shows that in the presence of the SOCD preplasma, at $200 \mathrm{~T}$ a proton beam with $\sim 460 \mathrm{MeV}$ peak energy and (FWHM) energy spread $\sim 33 \mathrm{MeV}$ is produced from the hydrogen layer. The monoenergetic peak of the beam is $\sim 4^{\circ}$ off the laser axis and its angular spread is $\sim 3^{\circ}$. For comparison, without the preplasma the peak energy of the protons is only about $340 \mathrm{MeV}$ and the energy spread is about $63 \mathrm{MeV}$. Moreover, in this case the beam would be double peaked in space and each peak also has large angular spread. The laser-to-energetic proton (i.e., only the monoenergetic part of the proton energy distribution is included) energy conversion efficiency is about $1 \%$, versus $0.7 \%$ without the preplasma, $0.1 \%$ in Ref. [17], and $10 \%$ in Ref. [29], but in the latter all protons are included in the estimate. That is, the long preplasma not only enhances the peak proton energy but also reduces its spread in both energy and space. That is, the characteristics of the energetic protons can to a certain degree be controlled by appropriately designing the preplasma, (see also Sec. IV). We note that, compared with the results for TNSA using underdense preplasma and lower intensity laser [30], the protons obtained here are of higher energy, more monoenergetic, and less angular spread. However, in the present case the entire target is destroyed by the intense LPI.

\section{ELECTRON DYNAMICS IN THE PREPLASMA}

We now consider in more detail the electron dynamics in the preplasma, which is crucial to the properties of the hot electron cloud behind the target. Since here $\tau \gg T$, the time averaged (over the light wave oscillations) laser ponderomotive force on an electron is [34]

$$
F_{p}=-4 m_{e} c^{2} \nabla\left|1+a^{2}\right|^{1 / 2}
$$

where $a=e E / m_{e} \omega c$. As mentioned, the affected electrons are driven forward as well as laterally outward by the ponderomotive force, creating a positively charged channel 

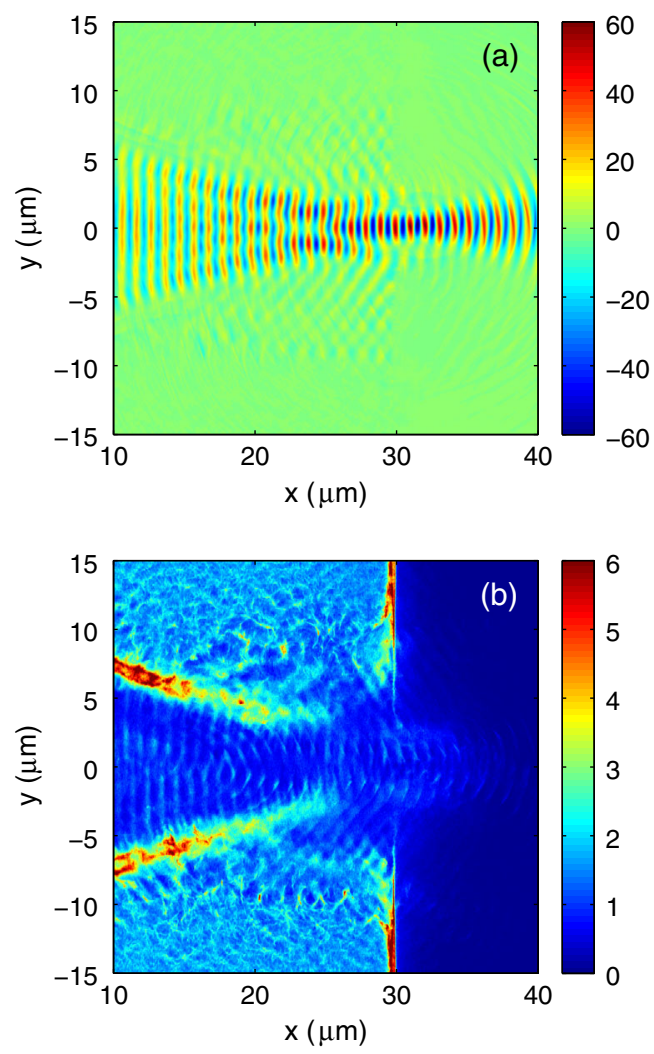

FIG. 2. (a) Wave electric field $E_{y}$ and (b) electron density in the carbon preplasma at $80 T$, shortly after the front of the selffocused laser pulse has passed through the target. For a better view of the electron dynamics, the main carbon and hydrogen layers are not shown. Here one can clearly see the preplasma electrons propagate with the laser field as periodic microbunched ( $\sim 1 \mu \mathrm{m}$ thick) sheets, and they also contribute to the target-back hot electron cloud.

with much reduced electron density. The laser light is also self-focused at the thin dense center target layer.

However, the low-density electrons remaining in the channel can also be self-consistently trapped and directly accelerated in the fields of the later part of the laser pulse. Figure 2 shows the wave electric field $E_{y}$ and the electron density. We see that the electrons in the laser-created lowdensity channel are trapped and bunched in the wave fields in a form of betatron oscillations. Such nonlinear waveelectron structures were also observed by Németh et al. [35] in their study of beam disparity in laser wakefield acceleration of electrons in low density plasmas. The microbunched electron sheets are of width $\lambda / 2$ and are in phase with the wave magnetic field. They propagate at the group velocity of the laser light in the channel and penetrate through the main carbon and the hydrogen layers to enhance the sheath field behind it. Since the lateral spread of the periodic electron sheets follows roughly that of the focused waist of the laser, the electron density enhancement immediately behind the target is mainly near

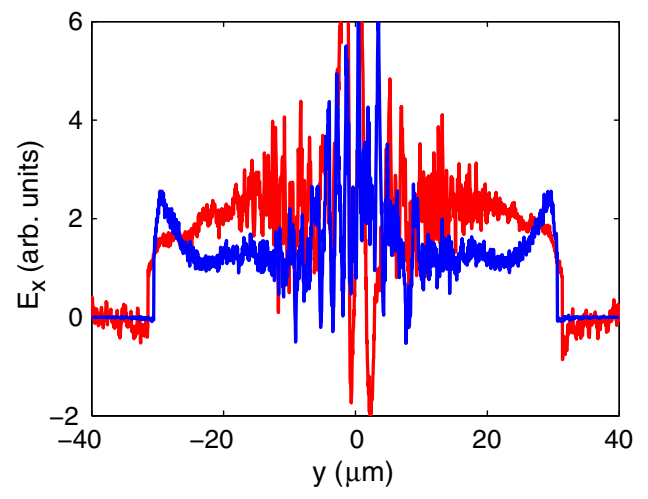

FIG. 3. The distribution of the axial electric field $E_{x}$ in the hydrogen layer at $t=80 T$ for with (red) and without (blue) the preplasma.

the axis, as can be seen in Fig. 2(b). Throughout the process the massive carbon ions in the preplasma are also affected by the LPI but the tend to remain in the channel during the time of interest here.

Figure 3 shows the transverse profile of the axial electric field component $E_{x}$ in the proton layer at $t=80 T$, where the red and blue curves are for with and without the preplasma, respectively. One can see that in the presence of the preplasma the averaged $E_{x}$ is stronger and more uniform. As a result, the accelerated protons are more monoenergetic, as shown in Fig. 1. As expected, we found that (not shown) increasing the thickness of the proton layer would make the TNSA protons less monoenergetic.

\section{PREPLASMA WITH LATERAL DENSITY PROFILE}

To see if the quality of the TNSA proton beams can be further improved, for example, by varying the lateral density profile, we now consider a preplasma with density profile $n=n_{0} \exp \left(-y^{2} / g^{2}\right)$, where $g$ is the characteristic width of the transverse density profile. The laser and other parameters are the same as in the preceding simulations for uniform preplasma.

Figure 4(a) shows that for $g=10 \lambda$, the peak TNSA proton energy is $489 \mathrm{MeV}$ at $t=200 T$, with an energy spread of $30 \mathrm{MeV}$. In fact, Fig. 4(b) shows that as $g$ is increased to $g=12 \lambda(=2 w)$, the maximum proton energy increases to $506 \mathrm{MeV}$, but decreases thereafter. The overall scenario of the process is similar to the case of uniform preplasma. As to be expected, in the LPI the same nonlinear effects as discussed earlier occur. However, because of the preplasma density profile, there are now less electrons on the periphery of the focused laser. Since the electrons at $y=w / 2$ encounter the maximum radial ponderomotive force, the axis region of the channel becomes more positive and the laser self-focusing becomes weaker. The resulting cross sections of the laser and accelerated preplasma electrons as they reach the main target are also larger. 

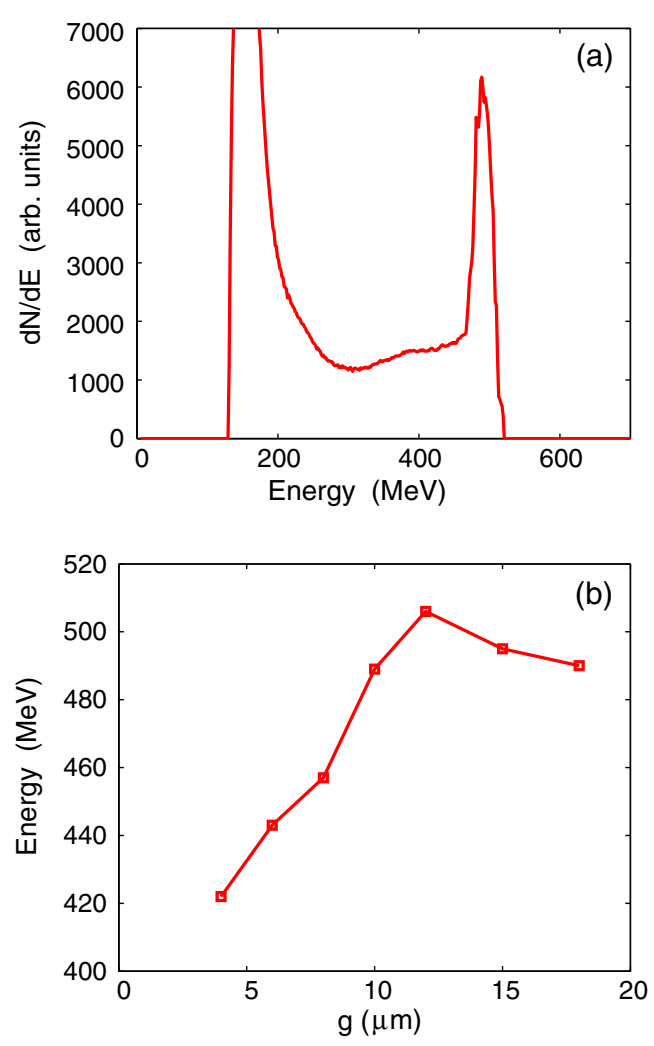

FIG. 4. (a) Spectrum of the TNSA protons behind the target at $t=200 T$ for $g=10 \lambda$ the width of the transverse density profile of the preplasma. (b)Peak proton energy versus $g$.

\section{CONCLUSION}

In this paper, we have considered TNSA of protons in the presence of a long SOCD preplasma, which supplies the hot electrons that create the space-charge field at the back of the target. The preplasma electrons are accelerated forward as well as driven sideways by the laser ponderomotive force, resulting in a channel. The electrons in the low-density channel are trapped by the laser fields and driven forward together with the latter, so that the targetback hot electron cloud is enhanced compared with that from TNSA without the long preplasma. As a result, higher proton energy with less energy and angular spread are achieved. We found that a proton bunch of $460 \mathrm{MeV}$ with $33 \mathrm{MeV}$ and $3^{\circ}$ spread is obtained, which is $35 \%$ higher and with less spread than that without the preplasma. The scheme can also be improved by using tailored preplasma. For example, with a laterally Gaussian preplasma density profile, one can even obtain $506 \mathrm{MeV}$ protons with $44 \mathrm{MeV}$ energy spread. Since our main concern here is to see the effect of the long preplasma and the production of a large number of monoenergetic protons, we have used a large thin hydrogen layer. In applications where highly localized energetic protons is needed, one can use smaller and denser hydrogen layers [7-11]. However, for cases where more accurate estimates are needed, 3D PIC simulations of the
TNSA process should be used since $2 \mathrm{D}$ results tend to overestimate the resulting proton energy and density $[5,29]$.

\section{ACKNOWLEDGMENTS}

This work is supported by the National Natural Science Foundation of China (NNSFC) under Grants No. 11475147, No. 11374262, and No. 61627901, and the Fundamental Research Fund for Chinese Central Universities under Grant No. 2018FZA3004.

[1] P. Gibbon, Short Pulse Laser Interactions With Matter: An Introduction (Imperial College Press, London, 2000).

[2] S. Atzeni and J. Meyer-ter-Vehn, The Physics of Inertial Fusion (Oxford University Press, New York, 2004).

[3] N. Naumova, T. Schlegel, V. T. Tikhonchuk, C. Labaune, I. V. Sokolov, and G. Mourou, Hole Boring in a DT Pellet and Fast-Ion Ignition with Ultraintense Laser Pulses, Phys. Rev. Lett. 102, 025002 (2009).

[4] E. Fourkal, B. Shahine, M. Ding, J. S. Li, T. Tajima, and C. $\mathrm{Ma}$, Particle in cell simulation of laser-accelerated proton beams for radiation therapy, Med. Phys. 29, 2788 (2002).

[5] P. Bolton, P. Londrillo, A. Sgattoni, M. Sumini, and G. Turchetti, Numerical Investigation of Optical Acceleration of Protons and Perspectives for Hadrotherapy. Università di Bologna report, available at http://www.physycom.unibo .it/aladyn_pic/docs/report-firb.pdf.

[6] M. Borghesi, D. H. Campbell, A. Schiavi, M. G. Haines, O. Willi, A. J. MacKinnon, P. Patel, L. A. Gizzi, M. Galimberti, R. J. Clarke, F. Pegoraro, H. Ruhl, and S. Bulanov, Electric field detection in laser-plasma interaction experiments via the proton imaging technique, Phys. Plasmas 9, 2214 (2002).

[7] E. L. Clark, K. Krushelnick, J. R. Davies, M. Zepf, M. Tatarakis, F. N. Beg, A. Machacek, P. A. Norreys, M. I. K. Santala, I. Watts, and A. E. Dangor, Measurements of Energetic Proton Transport through Magnetized Plasma from Intense Laser Interactions with Solids, Phys. Rev. Lett. 84, 670 (2000).

[8] S. P. Hatchett et al., Electron, photon, and ion beams from the relativistic interaction of Petawatt laser pulses with solid targets, Phys. Plasmas 7, 2076 (2000).

[9] S. C. Wilks, A. B. Langdon, T. E. Cowan, M. Roth, M. Singh, S. Hatchett, M. H. Key, D. Pennington, A. MacKinnon, and R. A. Snavely, Energetic proton generation in ultra-intense laser-solid interactions, Phys. Plasmas 8, 542 (2001).

[10] H. Schwoerer, S. Pfotenhauer, O. Jaeckel, K. U. Amthor, B. Liesfeld, W. Ziegler, R. Sauerbrey, K. W. D. Ledingham, and T. Esirkepov, Laser-plasma acceleration of quasimonoenergetic protons from microstructured targets, Nature (London) 439, 445 (2006).

[11] B. M. Hegelich, B. J. Albright, J. Cobble, K. Flippo, S. Letzring, M. Paffett, H. Ruhl, J. Schreiber, R. K. Schulze, and J. C. Fernandez, Laser acceleration of quasimonoenergetic MeV ion beams, Nature (London) 439, 441 (2006). 
[12] T. Esirkepov, M. Borghesi, S. V. Bulanov, G. Mourou, and T. Tajima, Highly Efficient Relativistic-Ion Generation in the Laser-Piston Regime, Phys. Rev. Lett. 92, 175003 (2004).

[13] T. Esirkepov, M. Yamagiwa, and T. Tajima, Laser Ion-Acceleration Scaling Laws Seen in Multiparametric Particle-in-Cell Simulations, Phys. Rev. Lett. 96, 105001 (2006).

[14] A. Henig, S. Steinke, M. Schnurer, T. Sokollik, R. Horlein, D. Kiefer, D. Jung, J. Schreiber, B. M. Hegelich, X. Q. Yan, J. Meyer-ter-Vehn, T. Tajima, P. V. Nickles, W. Sandner, and D. Habs, Radiation-Pressure Acceleration of Ion Beams Driven by Circularly Polarized Laser Pulses, Phys. Rev. Lett. 103, 245003 (2009).

[15] X. Q. Yan, C. Lin, Z. M. Sheng, Z. Y. Guo, B. C. Liu, Y. R. Lu, J. X. Fang, and J. E. Chen, Generating High-Current Monoenergetic Proton Beams by a CircularlyPolarized Laser Pulse in the Phase-StableAcceleration Regime, Phys. Rev. Lett. 100, 135003 (2008).

[16] Z. M. Zhang, X. T. He, Z. M. Sheng, and M. Y. Yu, Highdensity highly collimated monoenergetic $\mathrm{GeV}$ ions from interaction of ultraintense short laser pulse with foil in plasma, Phys. Plasmas 17, 043110 (2010).

[17] Z. M. Zhang, X. T. He, Z. M. Sheng, and M. Y. Yu, Highenergy monoenergetic protons from multistaged acceleration of thin double-layer target by circularly polarized laser, Phys. Plasmas 18, 023110 (2011).

[18] Z. M. Zhang, X. T. He, Z. M. Sheng, and M. Y. Yu, Hundreds $\mathrm{MeV}$ monoenergetic proton bunch from interaction of $10^{20-21} \mathrm{~W} / \mathrm{cm}^{2}$ circularly polarized laser pulse with tailored complex target, Appl. Phys. Lett. 100, 134103 (2012).

[19] L. O. Silva, M. Marti, J. R. Davies, R. A. Fonseca, C. Ren, F. S. Tsung, and W. B. Mori, Proton Shock Acceleration in Laser-Plasma Interactions, Phys. Rev. Lett. 92, 015002 (2004).

[20] M. S. Wei, S. P. D. Mangles, Z. Najmudin, B. Walton, A. Gopal, M. Tatarakis, A. E. Dangor, E. L. Clark, R. G. Evans, S. Fritzler, R. J. Clarke, C. Hernandez-Gomez, D. Neely, W. Mori, M. Tzoufras, and K. Krushelnick, Ion Acceleration by Collisionless Shocks in High-IntensityLaser-Underdense-Plasma Interaction, Phys. Rev. Lett. 93, 155003 (2004).

[21] M. Chen, Z. M. Sheng, Q. L. Dong, M. Q. He, S. M. Weng, Y. T. Li, and J. Zhang, Ion acceleration by colliding electrostatic shock waves in laser-solid interaction, Phys. Plasmas 14, 113106 (2007).

[22] L. Yin, B. J. Albright, B. M. Hegelich, K. J. Bowers, K. A. Flippo, T. J. T. Kwan, and J. C. Fernndez, Monoenergetic and $\mathrm{GeV}$ ion acceleration from the laser breakout afterburner using ultrathin targets, Phys. Plasmas 14, 056706 (2007).

[23] M. Kaluza, J. Schreiber, M. I. K. Santala, G. D. Tsakiris, K. Eidmann, J. Meyer-ter-Vehn, and K. J. Witte, Influence of the Laser Prepulse on Proton Acceleration in Thin-Foil Experiments, Phys. Rev. Lett. 93, 045003 (2004).

[24] J. T. Seo, S. H. Yoo, and S. J. Hahn, Effects of underdense preplasma on the energetic proton generation in ultraintense short laser pulse interaction with an overdense plasma slab, J. Phys. Soc. Jpn. 76, 114501 (2007).

[25] H. Y. Wang, X. Q. Yan, J. E. Chen, X. T. He, W. J. Ma, J. H. Bin, J. Schreiber, T. Tajima, and D. Habs, Efficient and stable proton acceleration by irradiating a two-layer target with a linearly polarized laser pulse, Phys. Plasmas 20, 013101 (2013).

[26] F. L. Zheng, S. Z. Wu, H. Zhang, T. W. Huang, M. Y. Yu, C. T. Zhou, and X.T. He, Preplasma effects on the generation of high-energy protons in ultraintense laser interaction with foil targets , Phys. Plasmas 20, 123105 (2013).

[27] S. X. Luan, W. Yu, M. Y. Yu, H. B. Zhuo, H. Xu, A. Y. Wong, J. W. Wang, B. F. Shen, and Z. Z. Xu, Target normal sheath acceleration of foil ions by laser-trapped hot electrons from a long subcritical-density preplasma, Phys. Plasmas 21, 123110 (2014).

[28] T. Nakamura, M. Tampo, R. Kodama, S. V. Bulanov, and M. Kando, Interaction of high contrast laser pulse with foam-attached target, Phys. Plasmas 17, 113107 (2010).

[29] A. Sgattoni, P. Londrillo, A. Macchi, and M. Passoni, Laser ion acceleration using a solid target coupled with a lowdensity layer, Phys. Rev. E 85, 036405 (2012).

[30] M. Passoni, A. Zani, A. Sgattoni, D. Dellasega, A. Macchi, I. Prencipe, V. Floquet, P. Martin, T. V. Liseykina, and T Ceccotti, Energetic ions at moderate laser intensities using foam-based multi-layered targets, Plasma Phys. Controlled Fusion 56, 045001 (2014).

[31] A. Pukhov and J. Meyer-ter-Vehn, Relativistic Magnetic Self-Channeling of Light in Near-Critical Plasma: ThreeDimensional Particle-in-Cell Simulation, Phys. Rev. Lett. 76, 3975 (1996).

[32] A. Pukhov and J. Meyer-ter-Vehn, Relativistic laser-plasma interaction by multi-dimensional particle-in-cell simulations, Phys. Plasmas 5, 1880 (1998).

[33] H. Y. Wang, C. Lin, Z. M. Sheng, B. Liu, S. Zhao, Z. Y. Guo, Y. R. Lu, X. T. He, J. E. Chen, and X. Q. Yan, Laser Shaping of a Relativistic Intense, Short Gaussian Pulse by a Plasma Lens, Phys. Rev. Lett. 107, 265002 (2011).

[34] B. Quesnel and P. Mora, Theory and simulation of the interaction of ultraintense laser pulses with electrons in vacuum, Phys. Rev. E 58, 3719 (1998).

[35] K. Németh, B. F. Shen, Y. L. Li, H. L. Shang, R. Crowell, K. C. Harkay, and J. R. Cary, Laser-Driven Coherent Betatron Oscillation in a Laser-Wakefield Cavity, Phys. Rev. Lett. 100, 095002 (2008). 